\title{
PRIMARY UMBILICAL ENDOMETROSIS
}

${ }^{1}$ Panti AA, ${ }^{1}$ Tunau KA, ${ }^{2}$ Agbo SP, ${ }^{3}$ Mohammed U, ${ }^{4}$ Omokanye $10,{ }^{1}$ Ugege B ${ }^{1}$ Department of Obstetrics and Gynaecology Usmanu Danfodiyo University Teaching Hospital Sokoto ${ }^{2}$ Department of Surgery Usmanu Danfodiyo University Teaching Hospital Sokoto ${ }^{3}$ Department of Pathology Usmanu Danfodiyo University Teaching Hospital Sokoto

${ }^{4}$ Department of Obstetrics and Gynaecology University Teaching Hospital Ilorin

\author{
Abstract \\ Background: Primary endometriosis is the presence of endometrial glands and tissues \\ outside the endometrial cavity. Primary umbilical endometriosis is a very rare entity.
}

Case: We reported a 40-year-old Para 2 plus 2 Alive 2 patient who presented with 2 year history of umbilical swelling with cyclical pain. The case was managed by simple excision and histopathological examination diagnosed it as a case of umbilical endometriosis.

Correspondence: Dr Abubakar A Panti Department of Obstetrics and Gynaecology, Usmanu Danfodiyo University Teaching Hospital Sokoto E-mail: kapanti2002@yahoo.co.uk.
Conclusion: Endometriosis should be considered as a differential diagnosis in cases of any umbilical pain or swelling in women of reproductive age group.

Keywords: Primary, Endometriosis, Umbilicus, Sokoto.

\section{INTRODUCTION}

Gindong ndometriosis is defined as presence of functional endometrial tissue or gland outside the uterine cavity ${ }^{1}$. Women with endometriosis present with characteristics signs and symptoms like dysmenorrhoea, dyspareunia, chronic pelvic pain or infertility.

Endometriosis is one of the most commonly encountered benign tumour in gynaecology. It develops predominantly in women during the reproductive age and regresses after menopause or ovariectomy. ${ }^{2}$ Endometriosis is described at most sites in the body and this raises the question of how endometriosis is disseminated. That no one mechanism could explain the dissemination of endometriosis to all sites resulted in multiple theories regarding its pathogenesis. ${ }^{3}$

Unlike pelvic endometriosis where laparoscopic visualization alone may suffice, the histologic presence of either endometrial glands or stroma or haemosiderin laden macrophages is essential for accurate diagnosis of extra pelvic disease. ${ }^{4}$ The incidence of umbilical endometriosis is estimated to be $0.5 \%$ to $1 \%$ of all the women with an extra genital endometriosis. ${ }^{5}$

We report a case of primary endometriosis managed by simple excision of the nodule with diagnostic confirmation on histology. This may be the first case report from Nigeria.

\section{CASE REPORT}

A 40-year-old Para 2 plus 2 Alive 2 presented with cyclical umbilical swelling and pain for 2 years. She however, reported that the nodule increased in size during menstruation with associated increase in the severity of the pain. She had a regular menstrual cycle every 30 days. The flow was normal. She did not experience any dysmenorrhoea, dyspareunia or pelvic pain. She was symptom free in between her cycles. She had no urinary or gastrointestinal symptoms. She was not on any form of contraception. No past history of surgical operations.

On examination, she had a body mass index of $22 \mathrm{~kg} / \mathrm{m}^{2}$. She was not pale, not icteric, no peripheral lymphadenopathy. Her vital signs were normal. The chest was clinically clear. On abdominal examination there was a nodular mass at the umbilicus on the right side measuring $2 \times 3$ $\mathrm{cm}$. The nodule was firm in consistency and tender (Figures 1\&2). The pelvic examination was normal.

Surgical excision of the nodule was performed after the patient's informed consent was obtained. The pre-operative haemogram and biochemistry were normal. The nodule was submitted for histology. The histological examination of the excised material showed macroscopically, an ellipse of tissue measuring $2.5 \times 1 \times 1 \mathrm{~cm}$ focally covered by Negroid skin. 
Microscopically, endometrial glands and surrounding stroma with haemorrhage areas were seen (Figure 2). She did well post operatively and was symptom free on follow up at three month.

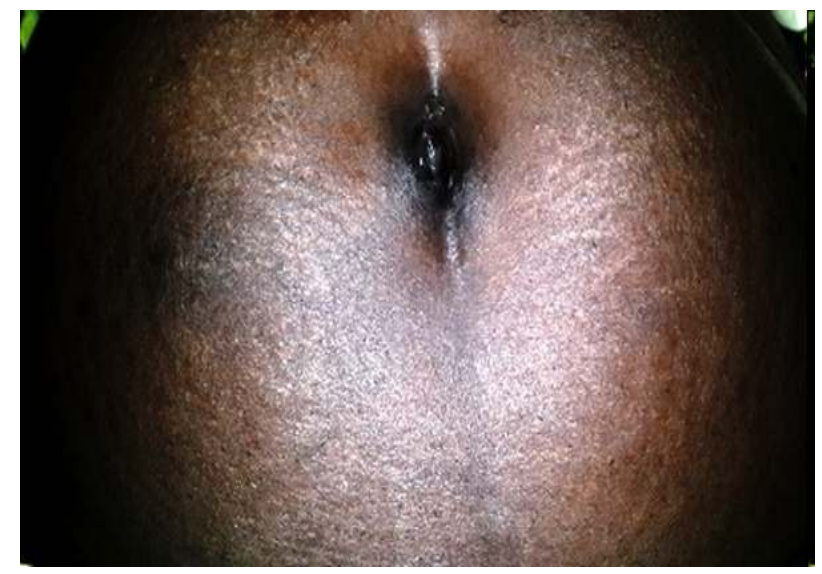

Figure 1: Umbilical endometriosis

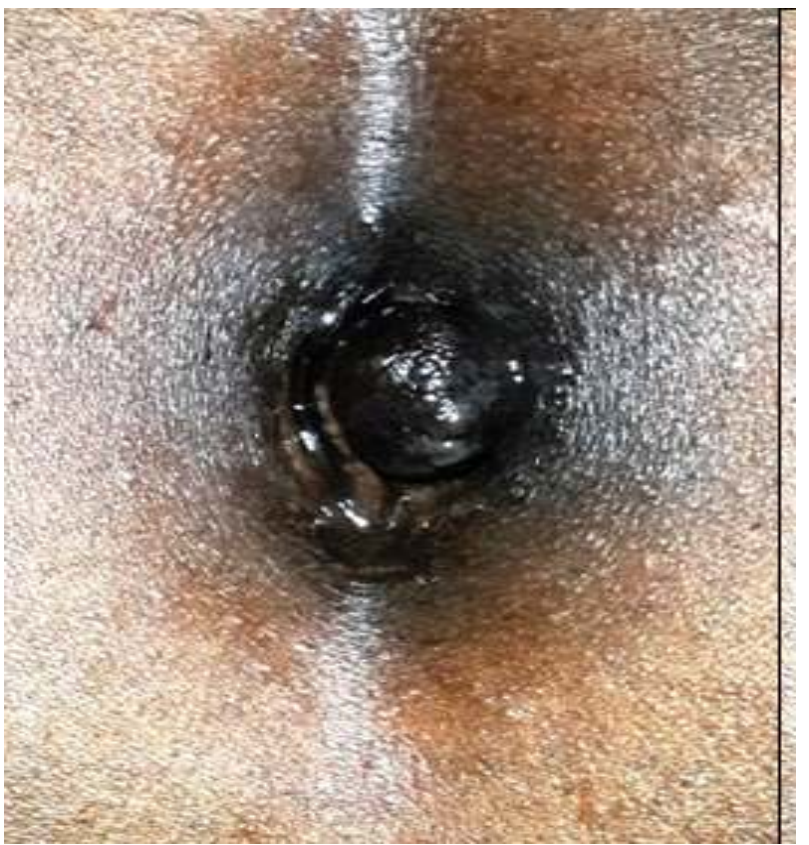

Figure 2: Umbilical nodule

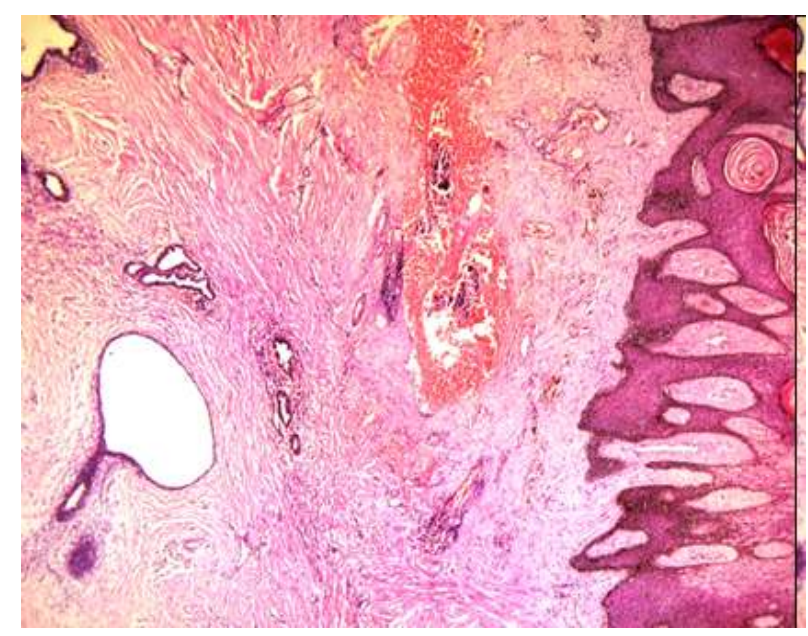

Figure 3: Histological photomicrograph of primary umbilical endometriosis

\section{DISCUSSION}

Endometriosis is the presence of functional endometrial tissue and glands outside the endometrial cavity. ${ }^{1}$ Endometriosis may be classified as secondary (scar) and primary (spontaneous), while the pathogenesis of spontaneous endometriosis is not clear, the pathogenesis accepted in scar endometriosis is the implantation of endometrial tissue to the skin. ${ }^{6}$

Primary umbilical endometriosis is very rare with an incidence of $0.5 \%$ to $1 \%$ of extra genital endometriosis. ${ }^{5}$ The most common symptoms of pelvic endometriosis are dysmenorrhoea, dyspareunia, pelvic pain, infertility and intestinal disorder. ${ }^{2}$ The case presented however, did not present with any of the above symptoms except for umbilical swelling and pain.

It appears clinically as nodular red- base or brown - black lesion ranging from a few millimetres to a few centimetres. The most characteristic symptom is cyclic pain together with a palpable mass. ${ }^{7}$ Just like was observed in the case presented. Surgical excision is the management of choice. The procedure could be performed under local anaesthesia aswas done for our patient.

There is no need to concurrently perform a diagnostic laparoscopy unless the patient has pelvic symptoms. Laparoscopy may increase morbidity and there is theoretical risk of disseminating the endometriosis from the umbilical port to the pelvis. ${ }^{8}$

\section{CONCLUSION}

In conclusion, we present a rare case of primary umbilical endometriosis especially in black race. The most characteristic symptom is cyclic pain together with a palpable mass. Umbilical endometriosis should be considered as differential diagnosis for any umbilical nodule or swelling in women of reproductive age group. 


\section{REFERENCES}

1. Adamson GD. Diagnosis and clinical presentation of endometriosis. Am J. ObsetGynecol 1990; 12:568-569

2. Nap AW, Grothius PG, Denis AY, Evers JLH, Duselman GAJ. Pathogenesis of endometriosis. Best Practice and ReserClin. ObstetGynaecol 2004;18(2): 33-244

3. Prentice A. Endometriosis. Current ObstetGynaecol 2002;12:155-160

4. Wanyonyi SZ, Sequeira E, Mukano G. Correlation between laparoscopic and histopathologic diagnosis of endometriosis. Int J. GynaecolObstet 2011;115:273-276
5. Latcher JW. Endometriosis of the umbilicus. GynecolObstet 1953;66:161163

6. Kyamida K, Lora V, Kamtakis J. Spontaneous cutaneous umbilical endometriosis: report of a new case with immunohistochemical study and literature review. Dermato online J 2011; 17(7):5

7. Skidmou RA, Wousley JT, Katz VI. Decidualised umbilical endometriosis. Int J. Surg 2010; 22: 85-86

8. Wanyonyi SZ, Gichere IH, Chege J. Primary umbilical endometriosis: case report JOGECA 2012; 24(1):26-27 